\title{
Impact of RNA-binding motif 3 expression on the whole transcriptome of prostate cancer cells: An RNA sequencing study
}

\author{
QINGZHUO DONG ${ }^{1}$, CHENGCHENG LV ${ }^{1}$, GEJUN ZHANG ${ }^{1,2}, \mathrm{ZI} \mathrm{YU}^{1,2}$, \\ CHUIZE $\mathrm{KONG}^{2}$, $\mathrm{CHENG} \mathrm{FU}^{1}$ and $\mathrm{YU} \mathrm{ZENG}^{1}$ \\ ${ }^{1}$ Department of Urology, Cancer Hospital of China Medical University, Liaoning Cancer Hospital and Institute, \\ Shenyang, Liaoning 110042; ${ }^{2}$ Department of Urology, The First Hospital of China Medical University, \\ Shenyang, Liaoning 110001, P.R. China
}

Received March 3, 2018; Accepted July 25, 2018

DOI: $10.3892 / o r .2018 .6618$

\begin{abstract}
RNA-binding motif 3 (RBM3) is a cold-shock protein that has been previously shown to attenuate cancer stem cell-like features in prostate cancer (PCa) cells. However, the mechanism underlying RBM3 regulation in PCa cells is largely unknown. The present study investigated the impact of RBM3 expression on the whole transcriptome of $\mathrm{PCa}$ cells using high-throughput RNA sequencing (RNA-seq). Differentially expressed genes (DEGs) that were identified through RNA-seq were applied to Gene Ontology (GO), pathway analysis, pathway-action networks and protein-protein interaction network analysis. GO and pathway ananlyses showed that RBM3 expression was associated with several metabolism pathways. Combining GO analysis and pathway analysis, certain DEGs, including phospholipase A2 group IIA (PLA2G2A), PLA2G2F, PLA2G4C, endothelin 1, cytochrome P450 family 2 subfamily B member $6, \mathrm{G}$ protein subunit $\gamma 5$, nitric oxide synthase 3 and CD38 molecule, were shown to be closely associated with RBM3 regulation in PCa cells. Furthermore, the changes in expression of selected genes upon RBM3-knockdown in RNA-seq were confirmed by separate reverse transcription-quantitative-polymerase chain reaction, validating the results of RNA-seq. Thus, the present study provides a series of valuable reference genes and pathways for the future study of the pathogenic role of RBM3 in the development of PCa.
\end{abstract}

Correspondence to: Professor Yu Zeng, Department of Urology, Cancer Hospital of China Medical University, Liaoning Cancer Hospital and Institute, 44 Xiaoheyan Road, Shenyang, Liaoning 110042, P.R. China

E-mail: zengyud@hotmail.com

Key words: RBM3, prostate cancer, RNA sequencing, transcriptome, PLA2G2A

\section{Introduction}

There were 4,292,000 new cases of cancer and 2,814,000 cases of cancer-associated mortality reported in 2015 in China (1). Among all types of cancer, prostate cancer ( $\mathrm{PCa}$ ) was diagnosed in $\sim 603,000$ men, and over 26,000 men succumbed to this disease (1). Although the incidence and mortality rates of PCa remain lower in China than in Western countries, they are increasing rapidly. Thus, $\mathrm{PCa}$ has become one of the major healthy issues for men in China.

RNA-binding motif 3 (RBM3) was originally found in the human fetal brain by Danno et al (2). The RBM3 gene is located at chromosome Xp11.23 and belongs to a highly conserved cold stress protein family. RBM3 was primarily reported as an RNA chaperone that preserves the translation of mRNAs at low temperatures. Recently, it was reported that the expression of RBM3 was also altered in response to other environmental stresses, including hypoxia, radiation, cellular apoptosis and necrosis (3). Additionally, RBM3 was found to be associated with brain development and may serve an important role in the differentiation of nerve cells (4). Although there have been a number of studies showing that RBM3 is upregulated in multiple cancer types, including ovarian cancer, breast cancer, melanoma, bladder cancer, colorectal cancer and PCa, the increased expression level of RBM3 in tumor samples was largely associated with a good prognosis (3,5-10). However, the role of RBM3 in PCa remains controversial. For example, Grupp et al (11) found that RBM3 was highly expressed in poorly differentiated and more invasive $\mathrm{PCa}$, and could be used as an independent prognostic factor to predict the early recurrence of tumors. On the other hand, Jonsson et al (10) reported that in PCa tissue samples from another patient cohort, the high expression of RBM3 protein, particularly RBM3 in the nucleus, was associated with a good prognosis, which may be due to the involvement of RBM3 in regulating a variety of cellular processes to maintain DNA integrity (10).

Our previous study found that the overexpression of RBM3 in PCa cells could suppress the expression of CD44 molecule (Indian blood group) (CD44) alternative splicing variant CD44v8-10; this suppression resulted in the cancer 
cells losing stem cell-like features, such as proliferating in soft agar or growing under the skin of nude mice. Thus, the loss of the expression of RBM3 enhances cell survival under stress and may favor the development of advanced cancer types (12). RBM3 may serve a different role in different types of cancer, and this possible role led to further investigation of the molecular relevance of RBM3 in PCa cells in the present study.

The present study analyzed the impact of RBM3 expression on the whole transcriptome of PCa cells using high-throughput RNA sequencing (RNA-seq). Through Gene Ontology (GO) analysis, pathway analysis and differentially expressed gene (DEG) interaction network analysis, the potential association between these DEGs and RBM3 regulation was investigated in an attempt to determine the molecular mechanisms underlying the impact of RBM3 on PCa development.

\section{Materials and methods}

Cell culture. The human prostate tumor PC 3 cell line was purchased from the American Type Culture Collection (ATCC; Manassas, VA, USA). Cells were cultured in RPMI1640 supplemented with $10 \%$ fetal bovine serum and $1 \%$ penicillin-streptomycin (all from HyClone; GE Healthcare Life Sciences, Logan, UT, USA) at $37^{\circ} \mathrm{C}$ in a humidified air incubator with $5 \% \mathrm{CO}_{2}$.

Plasmid and transfection. pCMV6-RBM3-GFP and pCMV6-AC-GFP, which were constructed previously (12), were transfected into PC3 cells at a final concentration of $1 \mu \mathrm{g} / \mathrm{ml}$ using Lipofectamine ${ }^{\circledR} 3000\left(\mathrm{P} 3000^{\mathrm{TM}}\right.$; Thermo Fisher Scientific, Inc., Waltham, MA, USA) according to the manufacturer's protocols. The cell medium was replaced with complete medium after $6 \mathrm{~h}$ of transfection, and the transfection efficiency was estimated $48 \mathrm{~h}$ later. The stably transduced PC 3 cells were selected by culturing cells in medium supplemented with puromycin for 20 days, and the PC3-RBM3\#1-GFP (PC3-R1) clone that overexpressed RBM3 and the PC3-AC-GFP (PC3-GFP) clone that was constructed as the control vector were chosen for further experiments.

Three pairs of short interfering RNAs (siRNAs) specific for RBM3 were synthesized by General Biosystems (Chuzhou, China). siRNA was transfected with PC-3 cells at $100 \mathrm{nM}$ total oligo concentration, using P3000 (Thermo Fisher Scientific, Inc.) according to the manufacturer's protocols. Briefly, the P3000 reagent and RNA were incubated for 5 min, and then the RNA-lipid complex was added to the cells. The cell medium was replaced with complete medium after $6 \mathrm{~h}$ of transfection, and the transfection efficiency was measured $48 \mathrm{~h}$ later. The siRNA with the highest knockdown efficacy was chosen for further experiments. All the subsequent experiments were performed within $48-72 \mathrm{~h}$ of transfection. The sequences of the siRNAs were as follows: si-RBM3-1 forward, 5'-CAUGUCCUCUGAAGAAGGAdT dT-3' and reverse, 5'-UCCUUCUUCAGAGGACAUGdT dT-3'; si-RBM3-2 forward, 5'-GGUUUCAUCACCUUCACC AdTdT-3' and si-RBM3-2 reverse, 5'-UGGUGAAGGUGA UGAAACCdTdT-3'; si-RBM3-3 forward, 5'-GAGACUAUA AUGGCAGAAAdTdT-3' and reverse, 5'-UUUCUGCCA UUAUAGUCUCdTdT-3'; si-NC forward, 5'-UUCUCCGAA
CGUGUCACGUdTdT-3' and reverse, 5'-ACGUGACACGUU CGGAGAAdTdT-3'.

Western blotting. Cells were harvested in radioimmunoprecipitation assay lysis buffer (Beyotime Institute of Biotechnology, Shenzhen, China) and heated for $10 \mathrm{~min}$ at $90^{\circ} \mathrm{C}$. Protein concentrations were measured using the bicinchoninic acid assay. A total of $50 \mu \mathrm{g}$ per lane of protein extracts was then separated by $10 \%$ SDS-polyacrylamide gel electrophoresis (SDS-PAGE) and transferred to polyvinylidene fluoride membranes (EMD Millipore, Billerica, MA, USA). The membranes were blocked with Tris-buffered saline plus Tween-20 (TBS-T; 0.1\% Tween-20) with 5\% (w/v) skimmed milk for $1 \mathrm{~h}$ at $37^{\circ} \mathrm{C}$ and then incubated with either RBM3 antibody (cat. no. AMAb90655; Atlas Antibodies AB, Stockholm, Sweden) that was diluted to $1: 1,000$ in TBS-T, or $\beta$-actin antibody (cat. no. A2228; Sigma-Aldrich; Merck KGaA, Damstadt, Germany) that was diluted to $1: 5,000$ in TBS-T, at $4^{\circ} \mathrm{C}$ overnight. Subsequent to being washed three times with TBS-T for 15 min each time, the membranes were incubated with the rabbit anti-mouse unconjugated $\operatorname{IgG}$ (cat. no. ab46540; Abcam, Cambridge, MA, USA) for $1.5 \mathrm{~h}$ at $37^{\circ} \mathrm{C}$. Subsequent to three washes with TBS-T for 15 min each time, the immuno bands were visualized using Enhanced Chemiluminescence reagents (Transgen Biotech, Co., Ltd., Beijing, China) on a MicroChemi $^{\mathrm{TM}}$ Chemiluminescent Imaging system (DNR Bio-Imaging Systems, Ltd., Mahale HaHamisha, Israel). $\beta$-actin (Sigma-Aldrich; Merck KGaA) was used as an internal control for total protein measurement.

RNA extraction and high-throughput sequencing. Total RNA was extracted using the SV total RNA Isolation kit (Promega Corporation, Madison, WI, USA) according to the manufacturer's protocols. The quality and quantity of the purified RNA were determined by measuring the absorbance at $260 / 280 \mathrm{~nm}$ using a Smartspec ${ }^{\mathrm{TM}}$ Plus spectrophotometer (Bio-Rad Laboratories, Inc., Hercules, CA, USA). RNA integrity was further verified by electrophoresis on a 1.5\% agarose gel.

For each sample, $10 \mu \mathrm{g}$ total RNA was used for RNA-seq library preparation using an RNA-Seq Library Preparation kit for Whole Transcriptome Discovery (Gnomegen, San Diego, CA, USA). Polyadenylated mRNAs were purified and concentrated with oligo(dT)-conjugated magnetic beads (Thermo Fisher Scientific, Inc.) prior to use for directional RNA-seq library preparation. Purified mRNAs were iron fragmented at $95^{\circ} \mathrm{C}$ followed by end repair and $5^{\prime}$-adaptor ligation. Next, reverse transcription was performed with reverse transcription (RT) primer harboring a 3'-adaptor sequence and randomized hexamer using RT Master mix (Gnomegen) with the following reaction conditions: $25^{\circ} \mathrm{C}$ for $10 \mathrm{~min}, 42^{\circ} \mathrm{C}$ for $40 \mathrm{~min}$ and $70^{\circ} \mathrm{C}$ for $15 \mathrm{~min}$, followed by cooling to $4^{\circ} \mathrm{C}$. The cDNAs were purified and amplified, and polymerase chain reaction (PCR) was performed using $2 \mathrm{X}$ HiFi PCR Master mix (Gnomegen) with the following reaction conditions: $98^{\circ} \mathrm{C}$ for $45 \mathrm{sec}, 98^{\circ} \mathrm{C}$ for $15 \mathrm{sec}, 60^{\circ} \mathrm{C}$ for $30 \mathrm{sec}$ and $72^{\circ} \mathrm{C}$ for $30 \mathrm{sec}$, for 15 cycles. PCR products corresponding to $200-500$ bps were collected by Size Selector (Gnomegen), and quantified and stored at $-80^{\circ} \mathrm{C}$ until used for sequencing.

For high-throughput sequencing, the libraries were prepared following the manufacturer's protocols and applied 
to an Illumina NextSeq 500 system (Illumina, Inc., San Diego, CA, USA) for $151 \mathrm{nt}$ pair-end sequencing by ABLife, Inc. (Wuhan, China).

$R T$-quantitative PCR (RT-qPCR). cDNA was synthesized using PrimeScript ${ }^{\mathrm{TM}}$ RT Master mix (Takara Biotechnology Co., Ltd., Dalian, China), and RT-qPCR was performed using SYBR Premix EX Taq ${ }^{\mathrm{TM}}$ (Takara, Dalian, China) and the LightCycler ${ }^{\mathrm{TM}} 480$ II system (Roche Diagnostics, Basel, Switzerland). PCR cycling conditions were $95^{\circ} \mathrm{C}$ for $30 \mathrm{sec}$, then $95^{\circ} \mathrm{C}$ for $5 \mathrm{sec}, 60^{\circ} \mathrm{C}$ for $30 \mathrm{sec}$ and $72^{\circ} \mathrm{C}$ for $30 \mathrm{sec}$, for 40 cycles. A housekeeping gene $\beta$-actin was used as the internal control for each gene. The primers for all genes were purchased from Sangon Biotech, Co., Ltd. (Shanghai, China). The sequences of the forward $(\mathrm{F})$ and reverse $(\mathrm{R})$ primers of selected genes were as follows (remainder are available upon request): PLA2G2A (F), 5'-ATGAAGACCCTCCTA CTGTTGG-3' and PLA2G2A (R), 5'-GCTTCCTTTCCT GTCGTCAACT-3'; GNG5 (F), 5'-ACTCAACCGCGTAAA AGTTTCC-3' and GNG5 (R), 5'-GGGTCTGAAGGGATT TGTACTTG-3'; EDN1 (F), 5'-AGAGTGTGTCTACTTCTG CCA-3' and EDN1 (R), 5'-CTTCCAAGTCCATACGGAACA A-3'; NOS3 (F), 5'-TGATGGCGAAGCGAGTGAAG-3' and NOS3 (R), 5'-ACTCATCCATACACAGGACCC-3'; GAL (F), 5'-CCGGCCAAGGAAAAACGAG-3' and GAL (R), 5'-GAG GCCATTCTTGTCGCTGA-3'; ACE (F), 5'-CCACGTCCC GGAAATATGAAG-3' and ACE (R), 5'-AGTCCCCTGCAT CTACATAGC-3'; CD38 (F), 5'-CAACTCTGTCTTGGCGTC AGT-3' and CD38 (R), 5'-CCCATACACTTTGGCAGTCTA CA-3'; MCHR2 (F), 5'-GCAGCAGCATTAACCCTTTTC-3' and MCHR2 (R), 5'-TCTCAGTCGCTCTTCTTTGGAT-3'; PTGDR2 (F), 5'-AAAAGGCTCGGGAAGGTTAAATG-3' and PTGDR2 (R), 5'-ACCGGGGAACCAAGAGAGAG-3'; RBM3 (F), 5'-CTTCAGCAGTTTCGGACCTA-3' and RBM3 (R), 5'-ACCATCCAGAGACTCTCCGT-3'; and $\beta$-actin (F), 5 -CATGTACGTTGCTATCCAGGC-3' and $\beta$-actin (R), 5'-CTCCTTAATGTCACGCACGAT-3'. The relative expression level (defined as a fold-change) of each target gene $\left(2^{-\Delta \Delta \mathrm{Cq}}\right)$ was normalized to the $\beta$-actin reference ( $\Delta \mathrm{Cq})(13)$. The final expression level of each gene was presented as the ratio of that in the experimental group to that in the control sample. Three independent experiments were performed and each sample was tested three times.

\section{Analysis of transcriptional data}

$G O$ analysis. In general, GO analyzes the following aspects: Integrating the protein group information from different organisms, determining the function of the protein domain; identifying the functional similarity of the abnormal expression of the gene in the disease or in aging; and predicting the genes associated with a disease. In the present study, GO analysis was performed to confirm the biological implications of the expression of specific genes in significant or representative profiles of genes that were differentially expressed, The data of the GO annotations, including the distribution of biological process (BP), cellular component (CC) and molecular function (MF) were downloaded from the GO Consortium (http://www.geneontology.org/) and the Database for Annotation, Visualization and Integrated Discovery (https://david.ncifcrf.gov/). Fisher's exact test was used to identify significant $\mathrm{GO}$ categories, and the false discovery rate (FDR) was used to correct the P-values.

Pathway analysis and pathway interaction network. Pathway analysis was used to confirm the significant pathways of the DEGs according to the Kyoto Encyclopedia of Genes and Genomes (KEGG) database (14), which is a database resource for understanding high-level functions and utilities of the biological system. Fisher's exact test was applied to select significant pathways, and the threshold of significance was defined according to the P-value and FDR. P $<0.05$ was determined to indicate a statistically significant difference. The unique genes enriched in those biological pathways with $\mathrm{P}<0.05$ (Fisher's exact test) were selected to be applied to ClueGO ver 2.3.3 (15) and the plug-in of Cytoscape ver 3.5.1 (16) to graphically represent pathways, namely the pathway interaction network. ClueGO is a Cytoscape plug-in that visualizes the biological terms for amount of genes in a functionally grouped network. In the network, the colour of the node could be switched between functional groups and cluster distribution easily.

Gene-action network. All the DEGs were analyzed to determine the association among them using the Search Tool for the Retrieval of Interacting Genes/Proteins (STRING; https://string-db.org/). STRING is a database of known and predicted protein-protein interactions (PPIs). The interaction association provided by STRING is mainly based on confidence score (reliability index) and other subsidiary information. The associations among the DEGs are included into the categories of 'neighborhood', 'experiment', 'databases', 'co-occurrence', 'co-expression', 'gene-fusion', 'textmining' and 'homology'. The STRING database was assigned according to the score of each directory, and then the combined score was drawn according to each assignment. The scores with a confidence interval of $>0.4$ were considered to be highly reliable and could represent the degree of interactions between DEGs.

Statistical analysis. Statistical analysis was performed using SPSS version 21.0 (IBM, Corp., Armonk, NY, USA). Experimental data are presented as the mean \pm standard deviation. Multigroup comparisons were conducted by one-way analysis of variance, and the difference was further analyzed by a Student-Newman-Keuls post hoc test. Pearson's correlation analysis was used to compare the correlation between the expression of genes in PC3-siR1 and PC3-siN cells. Fisher's exact test was applied to select significant altered pathways of DEGs. $\mathrm{P}<0.05$ was considered to indicate a statistically significant difference.

\section{Results}

Expression of RBM3 in cell lines. The expression of RBM3 in each cell line used in the study was detected by western blotting and RT-qPCR. As shown in Fig. 1, GFP-tagged RBM3 was strongly expressed in the RBM3-overexpressing PC3-R1 cell line, while endogenous RBM3 was markedly decreased in the si-RBM3-treated cells at the protein and mRNA levels. Among the three si-RBM3-treated cells, si-RBM3-1-treated cells (PC3-siR1) showed the largest decrease in the expression 



Figure 1. (A) mRNA expression of RBM3 was detected in each cell line by reverse transcription-quantitative polymerase chain reaction. RBM3 was highly expressed in PC3-R1, but downregulated in all siRBM3-treated PC3 cells compared with that in the control cells ("P<0.05 vs. PC3-siNC or PC3-GFP). (B) The protein level of RBM3 in each cell line was detected by western blotting. GFP-tagged RBM3 (43 kDa) expression was enhanced in PC3 cells (PC3-R1) that were stably transfected with pCMV6-RBM3-GFP compared with that in the control cells that were stably transfected with pCMV6-AC-GFP (PC3-GFP). At the same time, endogenous RBM3 protein $(17 \mathrm{kDa}$ ) was significantly decreased in the 3 si-RBM3-treated cells (PC3-siR1, PC3-siR2 and PC3-siR3) compared with that in the non-target siRNA-treated control cells (PC3-siNC). si, small interfering; NC, negative control; RBM3, RNA-binding motif 3.
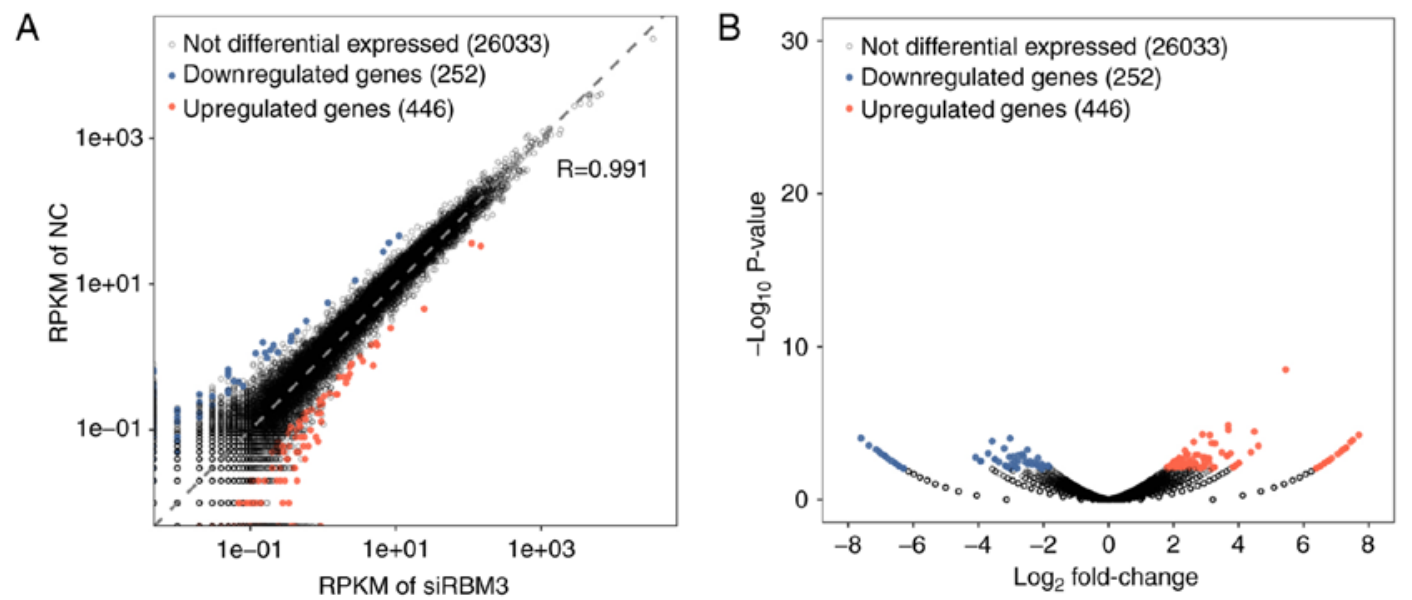

Figure 2. Identification of DEGs between PC3-siR1 cells and the control PC3-siNC cells. (A) Scatter plots represented all genes in two groups, and the global profiles of gene expression were generally highly correlated between PC3-siR1 and PC3-siNC cells (Pearson's correlation coefficient, R=0.991). (B) In the volcano plot, red dots represent DEGs that were upregulated, while blue dots represent DEGs that were downregulated (fold-change $\geq 2$ or $\leq 0.5$; P $\leq 0.05$ ). si, small interfering; NC, negative control; RBM3, RNA-binding motif 3; RPKM, reads per kilobase per million reads; DEGs, differentially expressed genes.

of RBM3. Thus, the PC3-siR1 cells were chosen for further RNA sequencing.

Display of RNA-seq analysis data. Total RNA isolated from PC3 cells treated with non-targeting control siRNA (PC3-siNC) or RBM3 siRNA (PC3-siR1) was subjected to RNA-seq. The decreased expression of RBM3 was verified in the sequencing data, and the high quality clean reads obtained in the sequencing data are available upon request.

GO analysis. The DEGs between the PC3-siNC and PC3-siR1 cells were screened out by edgeR. First, the data obtained in RNA-seq was evaluated for reliability. A total of 698 significantly DEGs were obtained; 446 genes were upregulated and 252 genes were downregulated in PC3-siR1 compared with that in PC3-siNC (Fig. 2). A volcano plot was created to show the overall changes in gene expression that were associated with RBM3-knockdown.

To further analyze the potential association between these DEGs and RBM3 regulation in PC3 cells, all the obtained DEGs were subjected to GOanalysis (Fig. 3). A total of 36 GO terms were enriched in BP layout among all the DEGs, including 'membrane depolarization', 'phosphatidylethanolamine acyl-chain remodeling', 'phosphatidylcholine acyl-chain remodeling', 'synaptic transmission' and 'blood circulation', among others (data not shown). Among the upregulated DEGs, 17 GO terms were enriched in BP layout, including 'multicellular organismal development', 'cartilage development', 'epidermis development', 'phototransduction', 'visible light' and 'cell differentiation', while among the downregulated DEGs, $10 \mathrm{GO}$ terms were enriched in BP layout, including 'cell-cell signaling', 'transport', 'transmembrane transport', 'synaptic transmission' and 'response to drug'. In the 36 GO terms among all the DEGs, several terms were closely associated with lipid metabolism, including 'glycerophospholipid biosynthetic process', 'phospholipid metabolic process', 'lipid catabolic process', 'phosphatidylethanolamine acyl-chain remodeling' and 'phosphatidylcholine acyl-chain remodeling'. The most associated genes were phospholipase A2 group IVC (PLA2G4C), PLA2G2A, PLA2G3, phospholipase B domain containing 1 and PLA2G2F. 

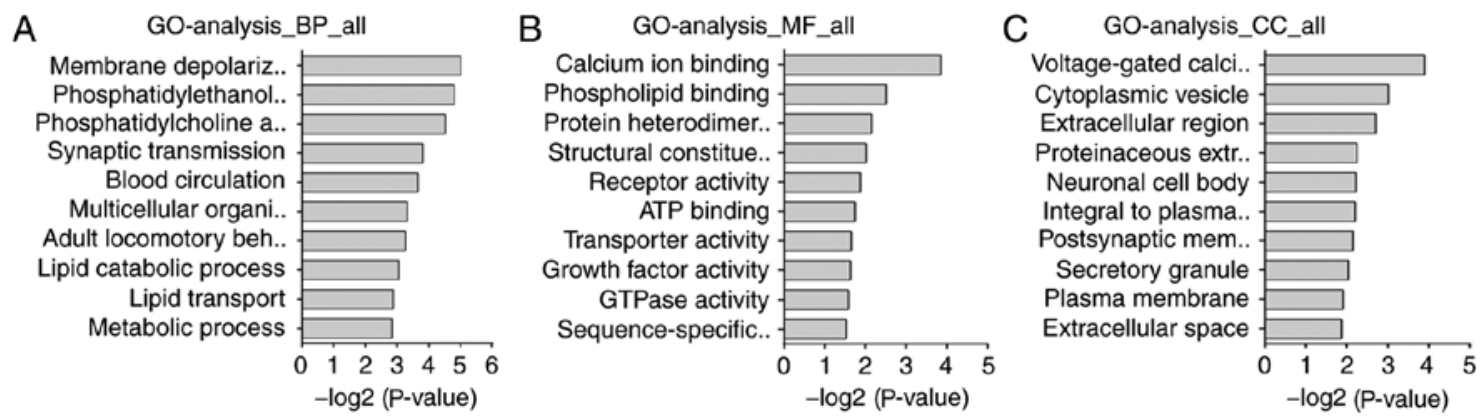

Figure 3. Significant GO terms from the PC3-siRBM3 cell lines. P $<0.05$ for all significant GO terms. (A) Significant GO terms on BP analysis. (B) Significant GO terms on MF analysis. (C) Significant GO terms on CC analysis. GO, Gene Ontology; si, small interfering; RBM3, RNA-binding motif 3; BP, biological process; $\mathrm{MF}$, molecular function; $\mathrm{CC}$, cellular component.

Table I. Top five enrichment signal pathways based on the Kyoto Encyclopedia of Genes and Genomes database for all differentially expressed genes.

\begin{tabular}{llccl}
\hline ID & \multicolumn{1}{c}{ Pathway } & Count & P-value & \multicolumn{1}{c}{ Genes } \\
\hline hsa04727 & GABAergic synapse & 6 & $1.00 \times 10^{-3}$ & CACNA1A,CACNA1B, GABRG3, GNG5,SLC38A5, \\
& & 5 & $1.40 \times 10^{-3}$ & SLC6A13 \\
hsa00565 & Ether lipid metabolism & 5 & PLA, PLA2G2F, PLA2G3, PLA2G4C, PLD1 \\
hsa04270 & Vascular smooth muscle contraction & 7 & $1.70 \times 10^{-3}$ & AVPR1B, GUCY1B3, NPR2, PLA2G2A, PLA2G2F, \\
& & & & PLA2G3, PLA2G4C \\
hsa05033 & Nicotine addiction & 4 & $2.20 \times 10^{-3}$ & CACNA1A, CACNA1B, GABRG3, GRIN3A \\
hsa04724 & Glutamatergic synapse & 6 & $3.20 \times 10^{-3}$ & CACNA1A, GNG5, GRIK2, GRIN3A, PLA2G4C, \\
& & & & PLD1
\end{tabular}

Pathway analysis. The KEGG database was applied to annotate the screened DEGs. The pathway terms that were significant based on Fisher's exact test $(\mathrm{P}<0.05)$ were screened.

A total of 362 genes were included in the pathway category analysis, and they were significantly enriched in 14 signal pathways, which were distributed in three main processes, namely, 'organismal systems', 'metabolism' and 'human diseases' (Fig. 4A). Next, a signal path interaction diagram (pathway interaction) was constructed through the ClueGO plug-in of Cytoscape (Fig. 4B). The entries with similar functions consisted of three major channel networks. The most abundant network included the pathways covering ether 'lipid metabolism', 'linoleic acid metabolism', 'alpha-linolenic acid metabolism', 'vascular smooth muscle contraction', 'pancreatic secretion' and 'fat digestion and absorption', while the other two major outlined networks were those containing the pathways of 'GABAergic synapse' and 'morphine addiction and nicotine addiction', and those containing the pathways of 'cardiac muscle contraction' and 'hypertrophic cardiomyopathy'. In addition, three pathways that are involved in 'glutamatergic synapse', 'hematopoietic cell lineage' and 'long-term depression' also partially intersected with the three major networks.

Notably, PLA2G2A, PLA2G2F, PLA2G3, PLA2G4C and phospholipase D1 (PLD1) were enriched in the cross points of several pathways, including 'ether lipid metabolism', 'linoleic acid metabolism', 'alpha-linolenic acid metabolism', 'pancreatic secretion' and 'fat digestion and absorption'.
Notably, these pathways are all closely associated with lipid metabolism, which has been shown to serve an important role in the development of PCa (17-19). The top five enrichment signal pathways for all DEGs are listed in Table I. Notably, the most listed DEGs, including PLA2G2A, PLA2G4C, PLA2G2F, $\gamma$-aminobutyric acid type A receptor $\gamma 3$ subunit, calcium voltage-gated channel subunit $\alpha 1 \mathrm{~B}$ (CACNA1B) and G protein subunit $\gamma 5$ (GNG5), are involved in multiple pathways, suggesting their important role in cellular processes.

PPI network based on DEGs. To further investigate the potential regulation of RBM3 on the transcriptome of PCa cells, a PPI network was constructed based on DEGs through the STRING online database (https://string-db.org/). Based on functional relevance, the associations of different genes in the database are described as 'neighborhood', 'experiment', 'databases', 'co-occurrence', 'co-expression', 'gene-fusion', 'textmining' and 'homology'. Scores were calculated based on the characteristic of each type of association. Thus, the weight of each association between two genes could be evaluated by the final comprehensive score. Only associated genes with scores of $>0.4$ were included in the network.

Notably, the majority of the upregulated DEGs that were identified in RNA-seq are closely associated with each other in the PPI network. As shown in Fig. 5, endothelin 1 (EDN1) and GNG5 have the highest degree nodes in the whole network, and they apparently locate centrally and have the most extensive associations with other DEGs. It is worth mentioning that 
A Pathway enrichment analysis of DEGs based on KEGG
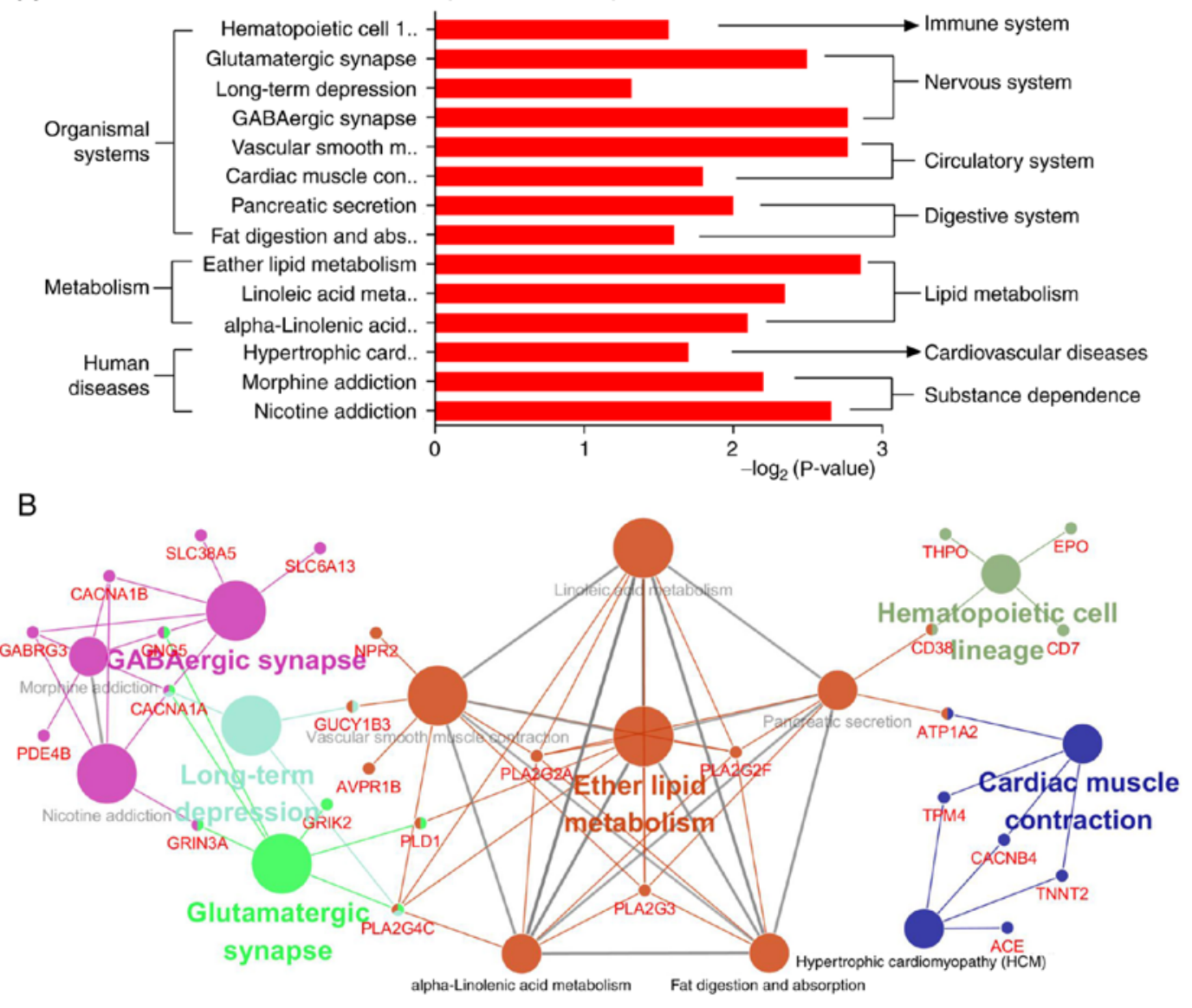

Figure 4. Pathway enrichment analysis of DEGs based on KEGG. (A) Enriched pathways of DEGs in PC3-siR1 cells compared to control PC3 cells; (B) Pathway-action network of DEGs in PC3-siR1 cells compared with that in control PC3 cells. si, small interfering; DEGs, differentially expressed genes; KEGG, Kyoto Encyclopedia of Genes and Genomes.

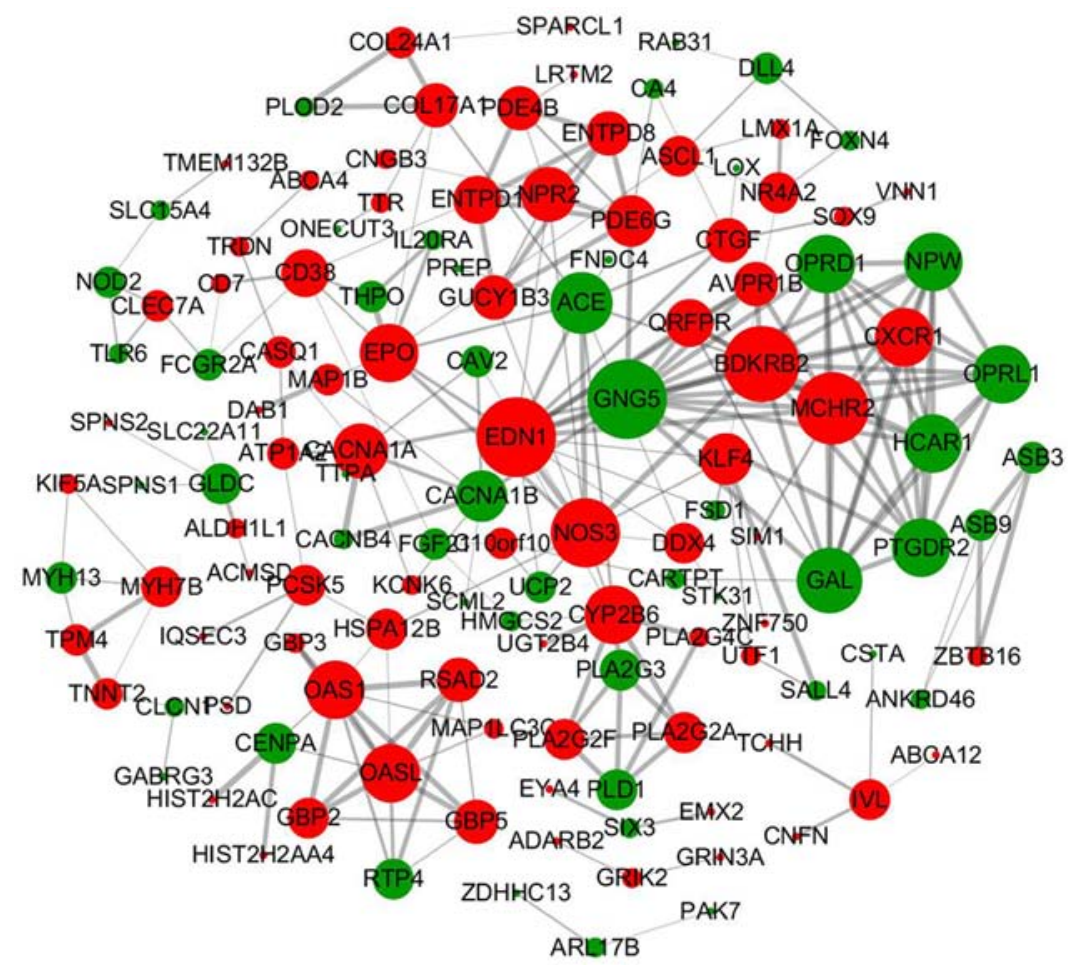

Figure 5. Protein-protein interaction network based on differentially expressed genes. The red dots represent the upregulated genes, while the green dots represent the downregulated genes. The size of the dots is increased with the PPI score, and the edges between genes represent interactions. The thickness of the edge represents the strength of the interaction. 



Figure 6. Changes in expression of certain DEGs, as confirmed by RT-qPCR. The expression changes of 21 DEGs upon RNA-binding motif 3-knockdown in RNA-seq were confirmed by qPCR ("P $<0.05$ vs. control). DEGs, differentially expressed genes; RT-qPCR, reverse transcription-quantitative polymerase chain reaction; si, small interfering; NC, negative control; RNA-seq, high-throughput RNA sequencing.

PLA2G2A, PLA2G2F and PLA2G3, which were frequently enriched in the pathway analysis or GO analysis, showed a close PPI as well, highlighting the fact that RBM3-knockdown significantly impacts the cellular network beyond isolated molecules. Similar interactions were observed among other DEGs, for example, natriuretic peptide receptor 2 (NPR2), ectonucleoside triphosphate diphosphohydrolase 1 (ENTPD1), ENTPD8, phosphodiesterase 6G (PDE6G), guanylate cyclase 1 soluble subunit $\beta 1$ (GUCY1B3) and PDE4B.

$R T-q P C R$ validation of the DEGs. The changes in the DEGs that were found by RNA-Seq were verified using RT-qPCR. The expression of 37 genes was examined in cell lines either overexpressing RBM3 or with RBM3-knockdown, as well as in control cells. Among the 37 genes, 21 were found to be associated with the expression levels of RBM3 exactly in the same pattern that had been shown in the RNA-seq, namely, either upregulated or downregulated upon RBM3-knockdown. The 18 genes that were upregulated by reducing RBM3 expression according to qPCR and RNA-seq were as follows: PLA2G2A, EDN1, nitric oxide synthase 3 (NOS3), CD38 molecule (CD38), 2'-5'-oligoadenylate synthetase-like (OASL), OAS1, guanylate binding protein 2, erythropoietin, radical S-adenosyl methionine domain containing 2, PLA2G2F, GUCY1B3, PDE6G, calcium/calmodulin-dependent protein kinase II inhibitor 1, glutamate ionotropic receptor kainate type subunit 2, troponin T2 cardiac type, Kruppel-like factor 4, PDE4B and connective tissue growth factor. At the same time, three genes were found to be downregulated in qPCR and RNA-seq. These genes were GNG5, galanin and GMAP prepropeptide and angiotensin I converting enzyme (Fig. 6). Moreover, there were another 10 genes, including receptor transporter protein 4, PLD1, 
hydroxycarboxylic acid receptor 1 , cytochrome P450 family 2 subfamily B member 6, CACNA1A, CACNA1B, ENTPD1, ENTPD8, SRY-box 9 and tropomyosin 4, showing the same trends as the RNA-seq data in cells expressing different levels of RBM3, but these differences were not statistically significant $(\mathrm{P}>0.05)$ (data not shown). Additionally, there were 6 genes that showed an inconsistent association with the expression of RBM3 between the qPCR and the RNA-seq experiments (data not shown). Taken together, using the separate RT-qPCR experiments, the changes in the majority of DEGs that were indicated in the RNA-seq could be confirmed; these genes may be representive of typical transcriptional regulation by RBM3 in the present PC3 cells and warrant further studies.

\section{Discussion}

The involvement of RBM3 in tumor development and progression has recently become a research focus. Accumulating data have shown that RBM3 is upregulated in various human tumors, but is associated with a good prognosis, although this finding remains controversial $(3,5-10)$. However, the association between RBM3 and PCa remains unexplored. This association prompted the use of high-throughput RNA sequencing in the present study in an attempt to profile the impact of RBM3 on the transcriptome of PCa cells.

It has been shown that the deregulation of important signaling pathways serves a central role in the development of cancer (20). In the present study, it was found that subsequent to knocking down RBM3, the expression of 698 genes involved in a number of important cancer-related signaling pathways was significantly altered. GO analysis showed that the DEGs were mainly enriched in tumor-related or nerve conduction-related signaling pathways, including 'membrane depolarization', 'phosphatidylethanolamine acyl-chain remodeling', 'synaptic transmission' and 'G-protein coupled receptor signaling pathway'. To further analyze the functional enrichment, all DEGs were input into each of the three categories, namely, BP, MF and CC. It was found that the pathways, including 'synaptic transmission', 'G-protein coupled receptor signaling pathway', 'elevation of cytosolic calcium ion concentration', 'signal transduction' and 'response to drugs' remained among the top pathways on the list. Consistent with other studies, RBM3 apparently participates in a variety of biological processes, including 'signal transduction', 'synaptic transmission' and 'drug reactions'.

To examine how RBM3 regulates pathways from another perspective, pathway analyses and the pathway interaction network were used. The significantly enriched signal pathway contained 14 items and 3 major networks. Notably, it was found that the pathways in the center of the major regulation network were all associated with metabolism. This finding is consistent with the results of GO_BP analysis, in which the 'lipid catabolic process' and the 'phospholipid metabolic process' were tightly associated with RBM3-regulation. This outcome suggests RBM3, as a cold-shock protein that mediates the stress response, may function through regulating a group of metabolic pathways.

The genes that were most frequently enriched in these networks include PLA2G2A, PLA2G2F, PLA2G3, PLA2G4C, PLD1, arginine vasopressin receptor 1B, GUCY1B3, NPR2, ATPase $\mathrm{Na}^{+} / \mathrm{K}^{+}$transporting subunit $\alpha 2$ and CD38. In particular, PLA2G2A, PLA2G2F, PLA2G3 and PLA2G4C are enriched repeatedly by multiple pathways. All these genes belong to the PLA2 family, in which the secretory PLA2G2A is the key enzyme of arachidonic acid metabolism and is involved in numerous pathophysiology processes (21), including inflammation, lipid metabolism, and phospholipid metabolism, as well as the regulation of insulin $(22,23)$. It has been shown that PLA2G2A is upregulated in LNCaP cells compared with certain other androgen-independent PCa cell lines. PLA2G2A protein was also found to be increased in the serum of PCa patients and is associated with high tumor stages and grades (24). Given that a low expression level of RBM3 was largely reported to be associated with a poor prognosis or aggressive progression in a number of cancer types, including $\mathrm{PCa}$, the increased PLA2G2A following RBM3-knockdown shown in the present study may favor tumor progression to a certain extent, and PLA2G2A may therefore be among the downstream effectors of RBM3-mediated regulation in cancer.

On the other hand, Su et al (25) found that PLA2G2A was involved in the astrocyte-mediated inflammatory response through a transcriptome study, indicating that PLA2G2A may be functionally associated with central nervous system homeostasis. Notably, it has been well documented that RBM3 serves a critical role in the neuroprotective effect of synaptic loss (4). In support of this claim, in the present study, pathways closely associated with synaptic transmission, including 'Glutamatergic synapse' and 'GABAergic synapse', were found to be associated with RBM3 regulation in pathway analysis and pathway interaction networks. Thus, in the present study, PLA2G2A and its family show their importance in mediating RBM3 regulation not only in cancer development, but also in neuroprotection. Further study of the regulation of RBM3 in a series of metabolic pathways, particularly those involving the PLA2 family, is clearly required.

In addition to metabolism and the PLA2 family, several other signaling pathways and gene families were found to be closely associated with RBM3 regulation in the present study; for example, GNG5 and the G protein pathway (26), EDN1 and the angiogenesis pathway (27), NOS3 and the NO synthesis pathway (28), CD38 and the signal transduction pathway (29), and CYP2B6 and the response to drug pathway (30). These pathways have all been reported to be important for cancer development. Thus, these particular gene families and signaling pathways may represent the potential regulatory network of RBM3 on cancer development and therefore warrant further study.

\section{Acknowledgements}

Not applicable.

\section{Funding}

This study was partially supported by the National Natural Science Foundation of China (grant nos. 81372766 and 81572532), and the Liaoning 'Climbing' scholarship.

\section{Availability of data and materials}

The datasets used and/or analyzed during the current study are available from the corresponding author on reasonable request. 


\section{Authors' contributions}

Conception and design: YZ and CF. Development of methodology: QD, CL and ZY. Analysis and interpretation of data (e.g., statistical analysis, biostatistics and computational analysis): QD, CL, ZY and GZ. Writing, review and/or critical revision of the manuscript: QD, CK and YZ. Administrative, technical or material support (i.e., reporting or organizing data, and constructing databases): QD, CK and CL. Study supervision: YZ and QD. All authors read and approved the final manuscript.

\section{Ethics approval and consent to participate}

Not applicable.

\section{Patient consent for publication}

Not applicable.

\section{Competing interests}

The authors declare that they have no competing interests.

\section{References}

1. Chen W, Zheng R, Baade PD, Zhang S, Zeng H, Bray F, Jemal A, Yu XQ and He J: Cancer statistics in China, 2015. CA Cancer J Clin 66: 115-132, 2016.

2. Danno S, Nishiyama H, Higashitsuji H, Yokoi H, Xue JH, Itoh K, Matsuda T and Fujita J: Increased transcript level of RBM3, a member of the glycine-rich RNA-binding protein family, in human cells in response to cold stress. Biochem Biophys Res Commun 236: 804-807, 1997.

3. Wellmann S, Truss M, Bruder E, Tornillo L, Zelmer A, Seeger K and Bührer C: The RNA-binding protein RBM3 is required for cell proliferation and protects against serum deprivation-induced cell death. Pediatr Res 67: 35-41, 2010.

4. Peretti D, Bastide A, Radford H, Verity N, Molloy C, Martin MG, MorenoJA,Steinert JR,Smith T, Dinsdale D, et al:RBM3 mediates structural plasticity and protective effects of cooling in neurodegeneration. Nature 518: 236-239, 2015.

5. Ehlén Å, Nodin B, Rexhepaj E, Brändstedt J, Uhlén M, Alvarado-Kristensson M, Pontén F, Brennan DJ and Jirström K: RBM3-regulated genes promote DNA integrity and affect clinical outcome in epithelial ovarian cancer. Transl Oncol 4: 212-221, 2011

6. Jögi A, Brennan DJ, Rydén L, Magnusson K, Fernö M, Stål O, Borgquist S, Uhlen M, Landberg G, Påhlman S, et al: Nuclear expression of the RNA-binding protein RBM3 is associated with an improved clinical outcome in breast cancer. Mod Pathol 22: 1564-1574, 2009.

7. Nodin B, Fridberg M, Jonsson L, Bergman J, Uhlén M and Jirström K: High MCM3 expression is an independent biomarker of poor prognosis and correlates with reduced RBM3 expression in a prospective cohort of malignant melanoma. Diagn Pathol 7: $82,2012$.

8. Florianova L, Xu B, Traboulsi S, Elmansi H, Tanguay S, Aprikian A, Kassouf W and Brimo F: Evaluation of RNA-binding motif protein 3 expression in urothelial carcinoma of the bladder: An immunohistochemical study. World J Surg Oncol 13: 317, 2015.

9. Melling N, Simon R, Mirlacher M, Izbicki JR, Stahl P, Terracciano LM, Bokemeyer C, Sauter G and Marx AH: Loss of RNA-binding motif protein 3 expression is associated with right-sided localization and poor prognosis in colorectal cancer. Histopathology 68: 191-198, 2016.

10. Jonsson L, Gaber A, Ulmert D, Uhlén M, Bjartell A and Jirström K: High RBM3 expression in prostate cancer independently predicts a reduced risk of biochemical recurrence and disease progression. Diagn Pathol 6: 91, 2011.
11. Grupp K, Wilking J, Prien K, Hube-Magg C, Sirma H, Simon R, Steurer S, Budäus L, Haese A, Izbicki J, et al: High RNA-binding motif protein 3 expression is an independent prognostic marker in operated prostate cancer and tightly linked to ERG activation and PTEN deletions. Eur J Cancer 50: 852-861, 2014.

12. Zeng Y, Wodzenski D, Gao D, Shiraishi T, Terada N, Li Y, Vander GDJ,Luo J, Kong C, Getzenberg RH, et al: Stress-response protein RBM3 attenuates the stem-like properties of prostate cancer cells by interfering with CD44 variant splicing. Cancer Res 73: 4123-4133, 2013.

13. Livak KJ and Schmittgen TD: Analysis of relative gene expression data using real-time quantitative PCR and the $2^{-\Delta \Delta C_{\mathrm{T}}}$ method. Methods 25: 402-408, 2001.

14. Kanehisa M and Goto S: KEGG: Kyoto encyclopedia of genes and genomes. Nucleic Acids Res 28: 27-30, 2000.

15. Bindea G, Mlecnik B, Hackl H, Charoentong P, Tosolini M, Kirilovsky A, Fridman WH, Pagès F, Trajanoski $\mathrm{Z}$ and Galon J: ClueGO: A Cytoscape plug-in to decipher functionally grouped gene ontology and pathway annotation networks. Bioinformatics 25: 1091-1093, 2009.

16. Shannon P, Markiel A, Ozier O, Baliga NS, Wang JT, Ramage D, Amin N, Schwikowski B and Ideker T: Cytoscape: A software environment for integrated models of biomolecular interaction networks. Genome Res 13: 2498-2504, 2003.

17. Ferro M, Terracciano D, Buonerba C, Lucarelli G, Bottero D, Perdonà S, Autorino R, Serino A, Cantiello F, Damiano R, et al: The emerging role of obesity, diet and lipid metabolism in prostate cancer. Future Oncol 13: 285-293, 2017.

18. Butler LM, Centenera MM and Swinnen JV: Androgen control of lipid metabolism in prostate cancer: Novel insights and future applications. Endocr Relat Cancer 23: R219-R227, 2016.

19. Al KO, Traka MH, Melchini A, Troncoso-Rey P, Jurkowski W, Defernez M, Pachori P, Mills RD, Ball RY and Mithen RF: Increased transcriptional and metabolic capacity for lipid metabolism in the peripheral zone of the prostate may underpin its increased susceptibility to cancer. Oncotarget 8: 84902-84916, 2017.

20. Yamamoto S, Tomita Y, Hoshida Y, Takiguchi S, Fujiwara Y, Yasuda T, Doki Y, Yoshida K, Aozasa K, Nakamura H, et al: Expression of hepatoma-derived growth factor is correlated with lymph node metastasis and prognosis of gastric carcinoma. Clin Cancer Res 12: 117-122, 2006.

21. Fijneman RJ and Cormier RT: The roles of sPLA2-IIA (Pla2g2a) in cancer of the small and large intestine. Front Biosci 13: 4144-4174, 2008.

22. Kuefner MS, Pham K, Redd JR, Stephenson EJ, Harvey I, Deng X, Bridges D, Boilard E, Elam MB and Park EA: Secretory phospholipase A2 group IIA modulates insulin sensitivity and metabolism. J Lipid Res 58: 1822-1833, 2017.

23. Six DA and Dennis EA: The expanding superfamily of phospholipase $\mathrm{A}_{2}$ enzymes: Classification and characterization. Biochim Biophys Acta 1488: 1-19, 2000.

24. Lu S and Dong Z: Overexpression of secretory phospholipase A2-IIa supports cancer stem cell phenotype via HER/ERBB-elicited signaling in lung and prostate cancer cells. Int J Oncol 50: 2113-2122, 2017.

25. Su Y, Wang Y, Zhou Y, Zhu Z, Zhang Q, Zhang X, Wang W, Gu X, Guo A and Wang Y: Macrophage migration inhibitory factor activates inflammatory responses of astrocytes through interaction with CD74 receptor. Oncotarget 8: 2719-2730, 2017.

26. Asano T, Shinohara H, Morishita R, Ueda H, Kawamura N, Katoh-Semba R, Kishikawa M and Kato K: Selective localization of $\mathrm{G}$ protein gamma5 subunit in the subventricular zone of the lateral ventricle and rostral migratory stream of the adult rat brain. J Neurochem 79: 1129-1135, 2001.

27. Rosanò L, Spinella F and Bagnato A: Endothelin 1 in cancer: Biological implications and therapeutic opportunities. Nat Rev Cancer 13: 637-651, 2013

28. Nikolić ZZ, Pavićević DLj, Romac SP and Brajušković GN: Genetic variants within endothelial nitric oxide synthase gene and prostate cancer: A meta-analysis. Clin Transl Sci 8: 23-31, 2015.

29. Benkisser-Petersen M, Buchner M, Dörffel A, Dühren-von-Minden M, Claus R, Kläsener K, Leberecht K, Burger M, Dierks C, Jumaa H, et al: Spleen tyrosine kinase is involved in the CD38 signal transduction pathway in chronic lymphocytic leukemia. PLoS One 11: e0169159, 2016.

30. Karakurt $\mathrm{S}$ and Adali O: Tannic acid inhibits proliferation, migration, invasion of prostate cancer and modulates drug metabolizing and antioxidant enzymes. anticancer agents Med Chem 16: 781-789, 2016. 